\title{
ON THE SINGULARITIES OF A CLASS OF FUNCTIONS ON THE UNIT CIRCLE
}

\author{
S. M. SHAH
}

Pólya has suggested and Szegö and others have proved the following theorem. ${ }^{1}$

THEOREM. Let $f(z)$ be a function regular in the whole plane including $z=\infty$ except at $z=1$. Let

$$
f(z)= \begin{cases}\sum a_{n} z^{n}, & |z|<1, \\ \sum b_{n} / z^{n}, & |z|>1 .\end{cases}
$$

If $a_{n}=O\left(n^{k}\right)$ and $b_{n}=O\left(n^{k}\right)$ then $f(z)$ is a rational function.

The above theorem is generalized in this paper as follows.

TheOREM 1. Let $f(z)$ be regular in the whole plane including $z=\infty$, except possibly at a certain set $S$ of points on $|z|=1$ (the set $S$ being not everywhere dense on the complete circumference of the unit circle). Let

$$
f(z)= \begin{cases}\sum a_{n} z^{n}, & |z|<1, \\ \sum b_{n} / z^{n}, & |z|>1,\end{cases}
$$

and let $a_{n}=O\left(n^{k}\right), b_{n}=O\left(n^{k}\right)$; then the following results hold.

(i) Every isolated singularity on $|z|=1$ will be a pole of order not exceeding $k+1$.

(ii) If there are only a finite number of singularities on $|z|=1$, then $f(z)$ is a rational function.

THeOREM 2. There exists a function satisfying the hypothesis of Theorem 1 and having an infinite number of singularities on the unit circle; also there exists a function satisfying the same hypothesis and having no isolated singularities.

LEMma 1. Let $f(z)$ be an integral function and let

$$
I_{p}(r)=\int_{0}^{2 \pi}\left|f\left(r e^{i \phi}\right)\right|^{p} d \phi \quad \text { where } p>0
$$

be bounded on a sequence of circles $r=r_{n}$ tending to infinity, for some $p>0$. Then $f(z)$ reduces to a constant.

Received by the editors November 28, 1945.

${ }^{1} \mathrm{~J}$. Deutschen Math. Verein vol. 40 (1931) Aufgaben und Lösungen p. 81 (Polya); ibid. vol. 43 (1934) Aufgaben und Lösungen pp. 13-16 (Szegö and others). 
Proof. $|f(z)|^{p}$ is subharmonic in any region of the z-plane. By Poisson's integral formula

$$
|f(z)|^{p} \leqq \frac{1}{2 \pi} \int_{0}^{2 \pi} \frac{\left(R^{2}-r^{2}\right)\left|f\left(R e^{i \phi}\right)\right|^{p} d \phi}{R^{2}+r^{2}-2 r R \cos (\phi-\theta)}
$$

where $|z|=r<R=r_{n}$. Hence

$$
|f(z)|^{p} \leqq \frac{1}{2 \pi} \frac{R^{2}-r^{2}}{(R-r)^{2}} \int_{0}^{2 \pi}\left|f\left(R e^{i \phi}\right)\right| p d \phi \leqq K \frac{R+r}{R-r} .
$$

Putting $R=2 r$ we get

$$
|f(z)|^{p} \leqq 3 K / 2 \text { on }|z|=R / 2 \text {. }
$$

Hence $f(z)$ is bounded on $|z|=r_{n} / 2$ and so it reduces to a constant.

LEMMA 2. Let $f(z)$ be regular for $|z| \geqq H$ except probably at infinity; and let

$$
\int_{0}^{2 \pi}|f(z)|^{p} d \phi
$$

be bounded on a sequence of circles $|z| r=r_{n}$ tending to infinity. Then $f(z)$ is regular at infinity.

Proof. We can write $f(z)=g(z)+h(z)$ where $g(z)$ is an integral function and $h(z)$ regular at infinity. Since $h(z)$ is bounded at infinity, it follows from Minkowski's inequality when $p>1$ (and still simpler when $p \leqq 1)$ that $\int_{0}^{2 \pi}|g(z)|^{p} d \phi\left(z=r e^{i \phi}\right)$ is bounded on a sequence of circles $r=r_{n}$ tending to infinity. Hence $g(z)$ is constant by Lemma 1 and so $f(z)$ is regular at infinity.

Proof of ThEOREM 1. To prove that every isolated singularity will be a pole, it is enough to prove that if $z=1$ is an isolated singularity, it is a pole since every other singularity can be brought to $z=1$ by a rotation. We suppose that $k$ is a positive integer. We have already supposed that $z=1$ is an isolated singularity of $f(z)$. Let $x=(1+z) /(1-z)$. This transforms the unit circle in the $z$-plane into the imaginary axis in the $x$-plane and $z=1$ corresponds to $x=\infty$. The function $\phi(x)=f(z)$ given by the above relation is therefore regular for $|x| \geqq R_{0}$ (where $R_{0}$ is some number), except at $x=\infty$.

From our assumptions about the coefficients we obtain

$$
|f(z)| \leqq \frac{c}{\left.|1-| z\right|^{k+1}}
$$

in the neighbourhood of the circle $|z|=1$. Hence 


$$
\left|(1-z)^{k+1} f(z)\right| \leqq c\left|\frac{1-z}{1-|z|}\right|^{k+1} .
$$

Let $\psi(x)=(1-z)^{k+1} f(z)$ where $z=(x-1) /(x+1)$. Then

$$
\begin{aligned}
|\psi(x)| & \leqq \frac{2^{k+1}}{|x+1|^{k+1}} \frac{c}{|1-|(x-1) /(x+1)||^{k+1}} \\
& \leqq \frac{c_{1}}{|| x+1|-| x-1||^{k+1}} \cdot
\end{aligned}
$$

Let $x=\rho e^{i \gamma}$. Then $|x+1|^{2}-|x-1|^{2}=4 \rho \cos \gamma$. Hence

$$
\begin{aligned}
|\psi(x)| & \leqq \frac{c_{1}\{|x+1|+|x-1|\}^{k+1}}{\left\{|| x+\left.1\right|^{2}-|x-1|^{2} \mid\right\}^{k+1}} \\
& \leqq c_{1}\left\{\frac{|x+1|+|x-1|}{4 \rho|\cos \gamma|}\right\}^{k+1} \\
& \leqq \frac{c_{2}}{|\cos \gamma|^{k+1}}
\end{aligned}
$$

if $\rho \geqq \rho_{0}$ is sufficiently large. Hence

$$
|\psi(x)|^{1 / 2(k+1)} \leqq \frac{c_{3}}{|\cos \gamma|^{1 / 2}}
$$

except when $\gamma= \pm \pi / 2$. Hence

$$
\int_{0}^{2 \pi}|\psi(x)|^{1 / 2(k+1)} d \gamma \leqq \int_{0}^{2 \pi} \frac{c_{3} d \gamma}{(|\cos \gamma|)^{1 / 2}}
$$

which is a convergent integral. Hence

$$
\int_{0}^{2 \pi}|\psi(x)|^{1 / 2(k+1)} d \gamma
$$

is bounded and so, by Lemma $2, \psi(x)$ is regular at infinity. Hence

$$
(1-z)^{k+1} f(z)
$$

is regular at $z=1$, which shows that $f(z)$ has a pole of order not exceeding $k+1$ at $z=1$.

This proves (i). To prove (ii) it follows by part (i) that each of the finite number of singularities on $|z|=1$ is a pole. Hence $f(z)$ is a regular function throughout the z-plane including infinity, except for a finite number of poles. Hence $f(z)$ is a rational function. 
Proof of Theorem 2. Consider the function

$$
f(z)=\sum_{1}^{\infty} \frac{1}{2^{n}} \frac{1}{\left(z-\alpha_{n}\right)}
$$

where $\left(\alpha_{n}\right)$ is any sequence of points on $|z|=1$. If the sequence $\left(\alpha_{n}\right)$ has only one limit point the above function $f(z)$ has a pole at each of these points $\alpha_{n}$ and an essential singularity at the limit point of the sequence $\left(\alpha_{n}\right)$. It is regular elsewhere. If

$$
f(z)=\sum_{0}^{\infty} a_{p} z^{p} \quad \text { for }|z|<1
$$

then

$$
a_{p}=-\sum_{n=1}^{\infty} \frac{1}{2^{n}} \frac{1}{\alpha_{n}^{p+1}}
$$

and therefore $\left|a_{p}\right| \leqq 1$. Similarly if $f(z)=\sum b_{p} / z^{p} \quad(|z|>1)$ then $\left|t_{p}\right| \leqq 1$. Hence $a_{p}$ and $b_{p}$ are certainly $O\left(n^{k}\right)$ for any $k \geqq 0$. To prove the second part, it is enough to take $\left(\alpha_{n}\right)$ in the above example to be everywhere dense on some arc of the unit circle, the arc not being the whole of the circumference. The function $f(z)$ will have a non-isolated essential singularity at every point of this arc and the coefficients $a_{n}$ and $b_{n}$ are bounded.

This example shows that the part (ii) of Theorem 1 is in a sense the best possible result, for the function constructed satisfies the conditions on the coefficients while it is not a rational function since it has an infinite number of singularities on the unit circle.

MusLim University 